\title{
Prussian blue nanocubes/carbon nanospheres heterostructure composite for biosensing of metformin
}

This article was published in the following Dove Press journal: International Journal of Nanomedicine

\author{
Jagriti Narang' \\ Nitesh Malhotra ${ }^{2}$ \\ Chaitali Singhal \\ Gajendra Singh ${ }^{3}$ \\ Chandra Shekhar Pundir ${ }^{4}$ \\ 'Amity Institute of Nanotechnology, \\ ${ }^{2}$ Amity Institute of Physiotherapy, \\ Amity University, Noida, Uttar \\ Pradesh, ${ }^{3}$ Department of Pharmacy, \\ University of Health Sciences, \\ ${ }^{4}$ Department of Biochemistry, M. D. \\ University, Rohtak, Haryana, India
}

Correspondence: Jagriti Narang Amity Institute of Nanotechnology, Amity University, Noida, Uttar Pradesh, India Email jags_biotech@yahoo.co.in

Nitesh Malhotra

Amity Institute of Physiotherapy, Amity

University, Noida, Uttar Pradesh, India

Email malhotra.nitesh@gmail.com

\begin{abstract}
This paper reports the fabrication of highly sensitive metformin sensor based on Prussian blue (PB) nanocubes/carbon nanosphere (CNS) heterostructures composed of a perfect cube and spherical composite on a fluorine-doped tin oxide surface. Due to the excellent biocompatibility of $\mathrm{PB}$ nanocubes the $\mathrm{PB} / \mathrm{CNS}$ based Mf sensor exhibited a wide linear range of $0.001-10 \mathrm{mM}$ with a response duration of less than $5 \mathrm{~s}$ and a detection limit (based on signal to noise ratio) of $0.1 \mu \mathrm{M}$.
\end{abstract}

Keywords: metformin, PB nanocubes, carbon nanospheres, sensor, FTO electrode

\section{Introduction}

Metformin (Mf) (500 mg Mf hydrochloride tablets) is used as the so-called anti-hyperglycemic drug. ${ }^{1,2}$ It causes some side effects, for instance, gastrointestinal irritation. ${ }^{3}$ Overdose of this medicine can also cause lactic acidosis, diarrhea, fast or shallow breathing, blurred vision, depression, and increased sweating. Therefore, there arises a need to identify selective and sensitive methods that determine the concentration of Mf. Though various methods are available for therapeutic drug monitoring of Mf, electrochemical techniques are the best alternatives because they are easy to operate, can be made available on-site for monitoring, need minimal sample preparation, and are sensitive. Different nanomaterials are attracting scientists toward infinite fields including biosensing because of their excellent properties such as sizes and shapes, the two factors that determine the performance of nanomaterials. Prussian blue (PB) is an exceptional material that can be employed in the fabrication of sensors, because of its non-toxicity, high electrocatalytic activity, and low overpotential detection. ${ }^{4}$ Carbon nanotubes and nanostructures have been broadly researched in the past few years. ${ }^{1,2}$ In this study, we used electrochemical sensing methods like voltammetry and electrochemical impedance spectroscopy for fast and sensitive detection of Mf.

\section{Materials and methods}

Preparation of PB nanocubes-carbon nanospheres (PBCNS heterostructures) on fluorine-doped tin oxide (FTO)

Kerosene oil was placed in a laboratory lamp and the lamp was lighted and allowed to burn in open air. An FTO electrode was placed above the flame of the lamp in order to collect the soot emitted from the lamps.

$\mathrm{CNS} / \mathrm{FTO}$ glass substrates were placed in a solution containing $\mathrm{K}_{3}\left[\mathrm{Fe}(\mathrm{CN})_{6}\right]$. $3 \mathrm{H}_{2} \mathrm{O}$ in $\mathrm{HCl}(0.01 \mathrm{M})$ solution was taken and continuously stirred for $1 \mathrm{~h}$. Then the 

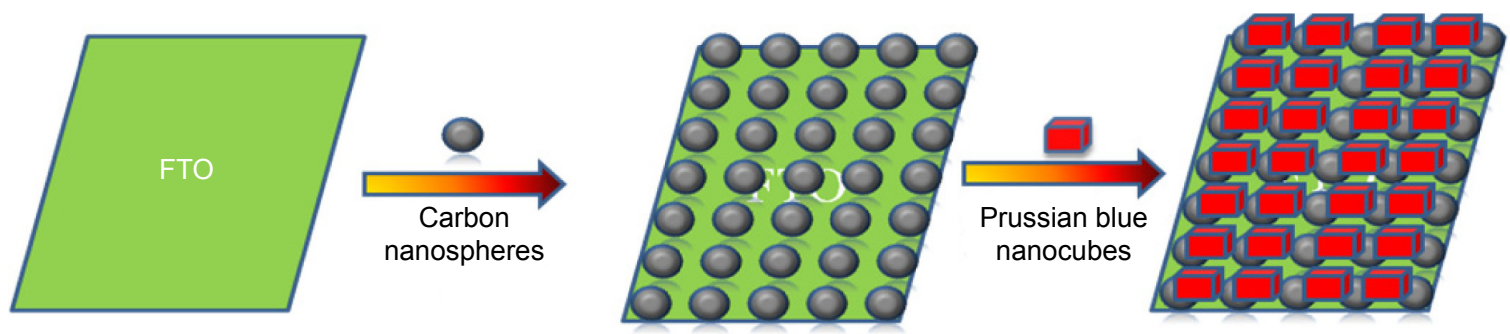

Scheme A Schematic representation showing the stepwise fabrication of a modified sensor. Abbreviation: FTO, fluorine-doped tin oxide.

beaker containing the CNS/FTO glass substrates was kept in an oven for dry coating. The PB-CNS heterostructure on FTO glass thus obtained was washed with ethanol and water thoroughly to remove the unadsorbed nanostructures on the FTO surface (Scheme A).

\section{Results and discussions}

Characterization of various stages of fabrication of PB-CNS heterostructures modified sensor

The general morphologies of the modified electrode (PBCNS heterostructures/FTO) were characterized by scanning electron microscopy (SEM). The SEM images showed that the diameters of the perfectly spherical shaped CNS were homogenous in nature (Figure 1A). However, it can be seen that the $\mathrm{PB}$ nanocubes were joined together through their bases with CNS, resulting in heterostructure (PB-CNS) morphologies (Figure 1B). The deposited CNS and PB-CNS heterostructure were also observed by X-ray diffraction patterns as shown in Figure 2. All the indexed peaks in the obtained pattern matched well with that of CNS and PB-CNS heterostructure. The XRD peaks of CNS in Figure 2A revealed two dominant peaks at 002 and 101 corresponding to CNS. The sharp and narrow peaks are indicative of the high crystalline structure of the spheres obtained. All the reflections can be readily indexed to PB-CNS in the XRD spectra in Figure 2B. The sharp narrow peaks clearly indicate the crystalline nature of the composites.
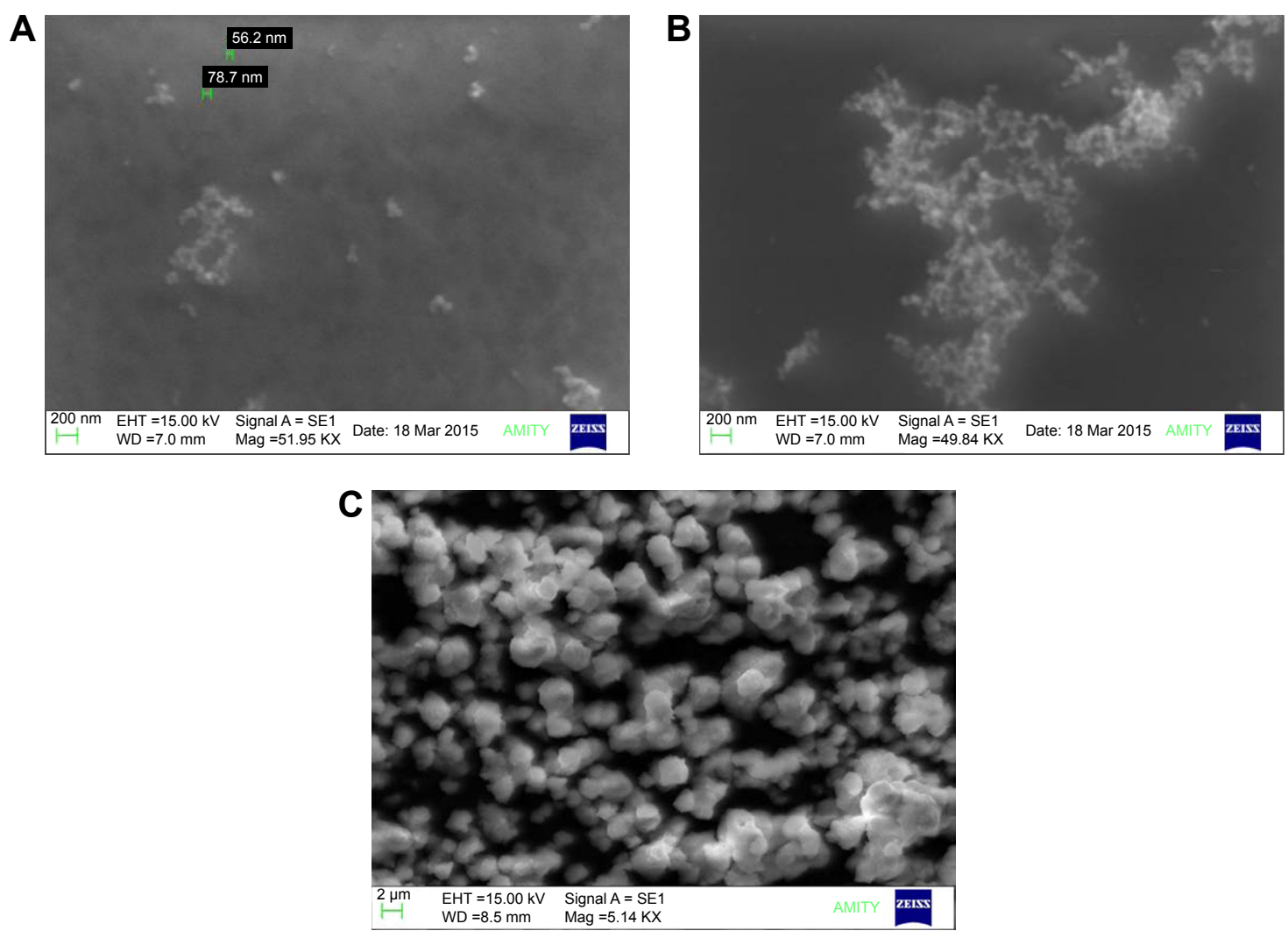

Figure I Scanning electron microscopy image of (A, B) carbon nanospheres (CNS), (C) PB-CNS/fluorine-doped tin oxide (FTO). 

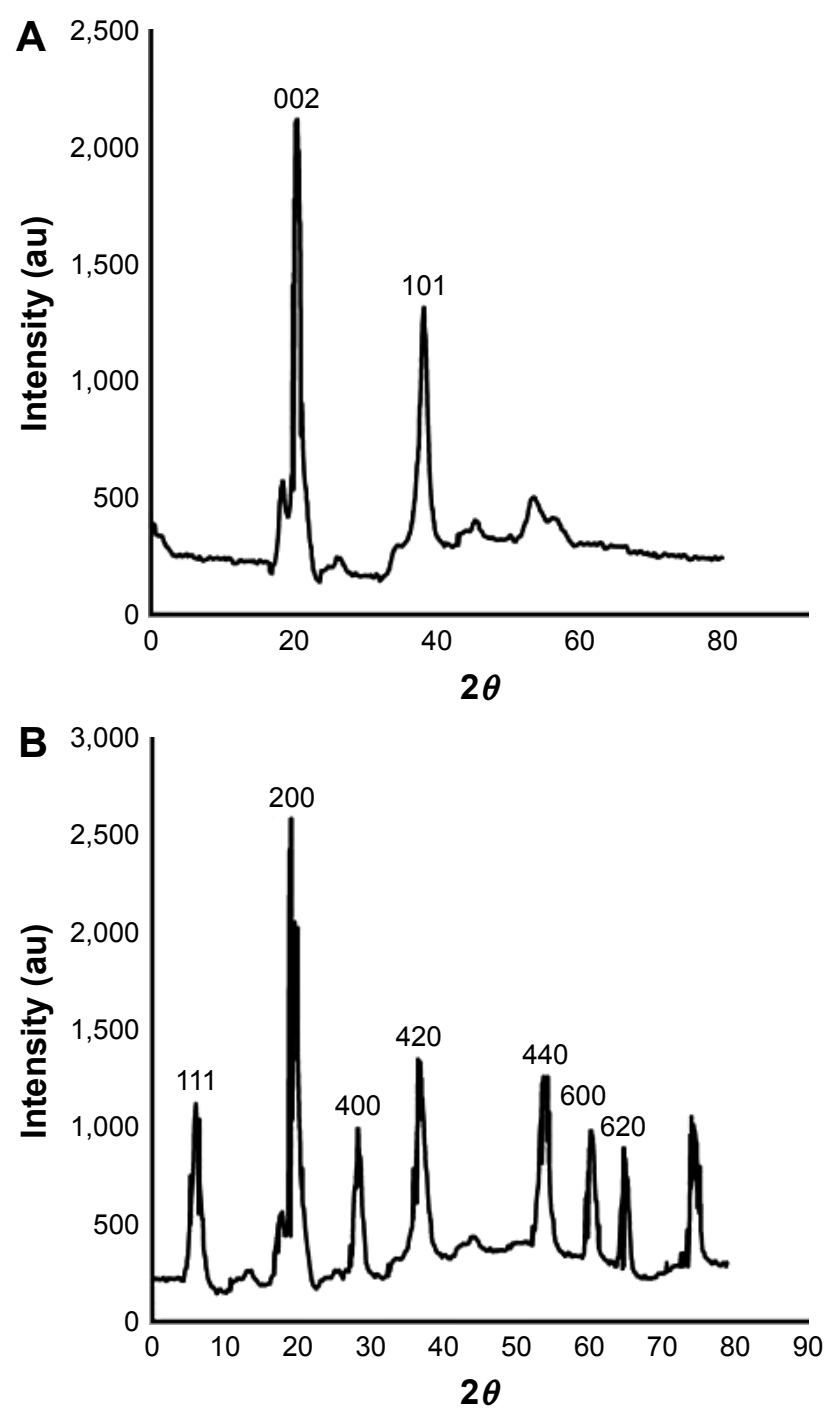

Figure 2 (A) X-ray diffraction pattern of carbon nanospheres (CNS) and (B) Prussian blue nanocubes-CNS (PB-CNS).

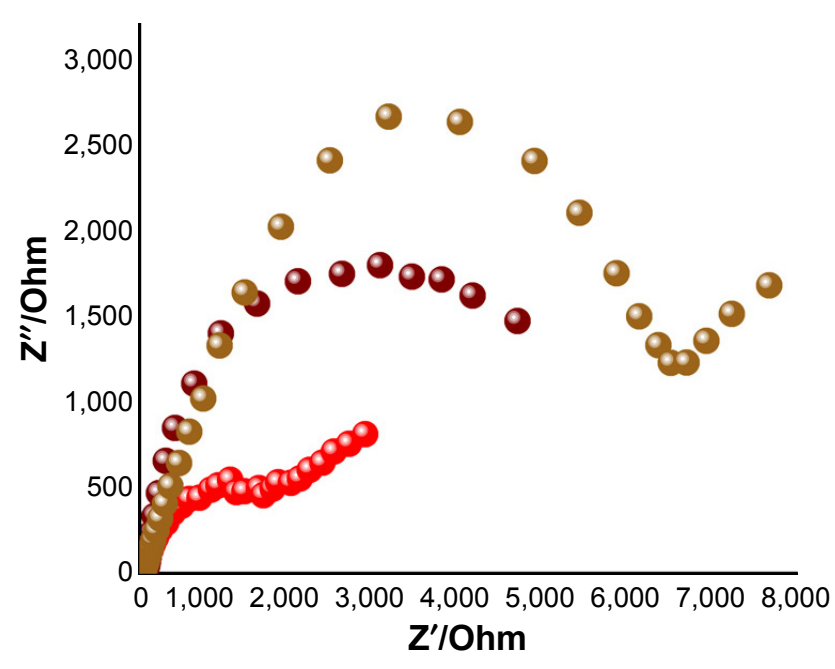

Figure 3 Electrochemical impedance spectroscopy of different stages of electrode.

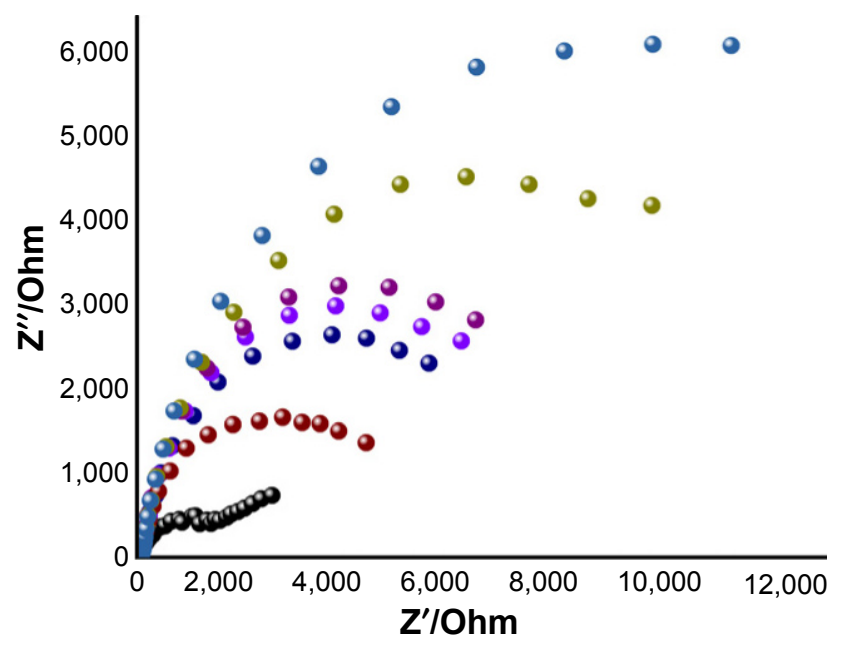

Figure 4 Impedimetric response of modified sensor using different concentrations of metformin.

Nyquist plots were obtained for FTO, CNS/FTO, and PB-CNS heterostructures/FTO electrodes, respectively, in a frequency range of $0.01-10 \mathrm{kHz}$ (Figure 3). It can be seen that the resistance charge transfer value for the FTO electrode $(6,800 \Omega)$ obtained is higher than that of the CNS/FTO electrode $(4,900 \Omega)$ and can be attributed to the conducting nature of CNS that promotes permeability of $\left[\mathrm{Fe}(\mathrm{CN})_{6}\right]^{3-4-4}$ to the electrode surface. After decoration of PB-CNS, the resistance charge transfer value further decreases to $1,000 \Omega$.

Detailed electrochemical response experiments have been carried out on the sensing PB-CNS heterostructures/FTO electrodes with the addition of different concentrations of $\mathrm{Mf}$ ranging from 0.001 to $10 \mathrm{mM}$ in $5 \mathrm{mM}\left[\mathrm{Fe}(\mathrm{CN})_{6}\right]^{3-} /\left[\mathrm{Fe}(\mathrm{CN})_{6}\right]^{4-}$ with an incubation time of $5 \mathrm{~s}$ (Figure 4). It is clearly seen from the spectra that as the concentrations of Mf increase, the charge transfer resistance decreases.

The sensors were evaluated by studying various parameters such as analytic recovery, precision, and accuracy of the proposed method. Analytic recovery of a known amount of added $\mathrm{Mf}$ was determined by the proposed biosensor. The mean analytic recoveries of Mf added at concentrations of 300 and $400 \mathrm{mM}$ were $99.5 \pm 1.4$ and $98.9 \pm 1.1$, respectively. The Mf content in ten spiked urine samples was estimated on a single day and five times again after storage at $-20^{\circ} \mathrm{C}$. The accuracy of the proposed method was $99 \%$.

\section{Conclusion}

In this study, we used PB nanocubes/CNS heterostructure composed of a perfect cube and spherical composite on FTO glass as a sensing platform for sensitive biosensing of Mf. The fabricated technique provided wide linear range (0.1-10 $\mathrm{mM})$ and anti-interference ability. 


\section{Disclosure}

The authors report no conflicts of interest in this work.

\section{References}

1. Clinical Guidelines Task Force, International Diabetes Federation. Glucose control: oral therapy. Global Guideline for Type 2 Diabetes. Brussels: International Diabetes Federation; 2005:35-38.

2. National Collaborating Centre for Chronic Conditions. Type 2 Diabetes: National Clinical Guideline for Management in Primary and Secondary care. London: Royal College of Physicians; 2008:86.
3. American Diabetes Association. Standards of medical care in diabetes 2009. Diabetes Care. 2009;32(Suppl 1):S13-S61.

4. Bolen S, Feldman L, Vassy J, et al. Systematic review: comparative effectiveness and safety of oral medications for type 2 diabetes mellitus. Ann Intern Med. 2007;147(6):386-399.

\section{Publish your work in this journal}

The International Journal of Nanomedicine is an international, peerreviewed journal focusing on the application of nanotechnology in diagnostics, therapeutics, and drug delivery systems throughout the biomedical field. This journal is indexed on PubMed Central, MedLine, CAS, SciSearch ${ }^{\circledR}$, Current Contents ${ }^{\circledR} /$ Clinical Medicine,
Journal Citation Reports/Science Edition, EMBase, Scopus and the Elsevier Bibliographic databases. The manuscript management system is completely online and includes a very quick and fair peer-review system, which is all easy to use. Visit http://www.dovepress.com/ testimonials.php to read real quotes from published authors.

Submit your manuscript here: http://www.dovepress.com/international-journal-of-nanomedicine-journal 